ORIGINAL ARTICLE

\title{
Effect of a fourth Haemophilus influenzae type $b$ immunisation in preterm infants who received dexamethasone for chronic lung disease
}

\author{
P Clarke, P J Powell, D Goldblatt, M J Robinson
}

Arch Dis Child Fetal Neonatal Ed 2003;88:F58-F61

See end of article for authors' affiliations

......................

Correspondence to: Dr P Clarke, Neonatal Intensive Care Unit, Hope

Hospital, Salford, UK paul.clarke@srht.nhs.uk

Accepted 16 July 2002

\begin{abstract}
Aim: To assess whether a fourth Hib polysaccharide-tetanus protein conjugate vaccine (PRP-T) would improve antibody response in preterm infants previously treated with dexamethasone for chronic lung disease.

Methods: In a pilot study 12 infants born at less than 30 weeks gestation who had received corticosteroids were given a supplementary Hib dose six weeks after completion of the primary immunisation course. Serum samples obtained before and at eight weeks following the fourth Hib dose were analysed for total level and avidity of anti-PRP antibody.

Results: There was no significant increase in the geometric mean titre (GMT) of anti-PRP antibody resulting from the fourth Hib immunisation (GMT: pre $2.35 \mu \mathrm{g} / \mathrm{ml}$, post $2.24 \mathrm{\mu g} / \mathrm{ml}, \mathrm{p}=0.79$ ). A subgroup of six infants had subprotective antibody levels $(<1.0 \mu \mathrm{g} / \mathrm{ml})$ after the primary immunisation course, which remained subprotective following the extra Hib immunisation. Despite the poor response in total antibody level, the study group showed a significant rise in PRP specific lgG avidity following the fourth immunisation (GMAl: pre 0.076, post 0.138, $p=0.043$ ).

Conclusion: An additional Hib immunisation given to recently steroid treated preterm infants six weeks after completion of the primary schedule did not augment primary immunogenicity. However, increasing avidity may imply successful priming and long term immunity to Hib.
\end{abstract}

U ntil recently, Haemophilus influenzae type b (Hib) was the most common cause of serious invasive infection in young children, ${ }^{1}$ with highest attack rates in infants less than 12 months of age. ${ }^{2}$ In term infants Hib conjugate vaccines are of proven efficacy. ${ }^{3}$ Successful Hib immunisation is defined as achievement of an antibody titre over 0.15 or 1.0 $\mu \mathrm{g} / \mathrm{ml}$ with the demonstration that immune memory has been generated. Although subject to equivalent Hib vaccines and schedules as term infants, only a handful of small studies have analysed rates of successful immunisation in preterm infants. Published data indicate an impaired antibody response to primary immunisation with the conjugate Hib vaccines PRP-T (polyribosylribitol phosphate-tetanus protein) and PRP-OMP (polyribosylribitol phosphate-outer membrane protein of meningococcus B), and with a newer combined Hib vaccine.$^{5-9}$ Extreme prematurity below 30 weeks gestation correlates with greater attenuation of antibody response. ${ }^{67}$ Only two studies describe preterm infants' antibody response to the accelerated Hib immunisation schedule used in the UK. Infants less than 32 weeks gestation showed impaired immunogenicity to three PRP-T doses if steroid treated ${ }^{8}$ and to PRP-T in a combined vaccine irrespective of steroid treatment. ${ }^{9}$

Extreme prematurity and corticosteroid treatment appear to be major factors attenuating antibody response to routine Hib immunisation in infants born preterm. It has been suggested that preterm infants may require extra doses of vaccine in order to attain adequate Hib titres. ${ }^{9}$ This study sought to analyse the immune response of steroid treated extremely preterm infants to an additional Hib vaccine dose. Our primary hypothesis was that giving a supplementary Hib dose six weeks following primary immunisations would boost the antibody response in steroid treated preterm infants. Antibody avidity was assayed as a secondary outcome measure in order to determine whether the booster immunisation resulted in a qualitative improvement in Hib antibody.

\section{METHODS}

\section{Patients}

The study commenced in February 1997; infants born at less than 30 weeks gestation were eligible if they had received at least one course of postnatal dexamethasone in the management of chronic lung disease (CLD). The participating centres were Hope, Royal Bolton, and Wythenshawe Hospitals. Fifteen infants were eligible during the 19 month recruitment period; all were enrolled following local ethics committee approval and informed parental consent. A pre-fourth Hib serum sample was available for 12 infants (six male, six female); 11 of these completed the study. Three infants without pre-booster serology were excluded. The median gestational age was 25 weeks (range 24-29 weeks); median birth weight was $830 \mathrm{~g}$ (range 550-1235 g). No infant received intravenous gammaglobulin therapy. All infants received at least one transfusion with SAG-M blood during their hospital stay, but in all instances transfusion occurred prior to completion of the primary immunisation schedule.

\section{Immunisations}

Infants received a primary Hib course along with DTP (adsorbed diphtheria, tetanus, and pertussis) vaccine at 2, 3 , and 4 months postnatal age in line with current recommendations..$^{10} \mathrm{~A}$ fourth Hib dose was administered six weeks following the third immunisation. The vaccine used was PRP-T (ActHIB; Pasteur-Mérieux-MSD, Lyon, France).

Abbreviations: $\mathrm{Al}$, avidity index; CLD, chronic lung disease; GMT, geometric mean titre; Hib, Haemophilus influenzae type b; PRP polyribosylribitol phosphate; PRP-OMP, polyribosylribitol phosphate-outer membrane protein of meningococcus B; PRP-T, Hib polysaccharide-tetanus protein conjugate vaccine 
Table 1 Timing of immunisations and serum samples

\begin{tabular}{lc}
\hline & $\begin{array}{l}\text { Age in days } \\
\text { Median (IQR) }\end{array}$ \\
\hline First Hib immunisation & $65(60-89)$ \\
Second Hib immunisation & $97(92-121)$ \\
Third Hib immunisation & $143(127-170)$ \\
First blood sample and fourth Hib immunisation & $205(164-218)$ \\
Second (post-fourth Hib) blood sample & $255(229-274)$ \\
Days between fourth Hib and second blood & $54(31-59)$ \\
sample & \\
\hline
\end{tabular}

Each $0.5 \mathrm{ml}$ dose contained $10 \mu \mathrm{g}$ of Hib capsular polysaccharide (polyribosylribitol phosphate, PRP).

\section{Steroids}

Antepartum steroids had been administered in each case. The initial dose of dexamathasone used in treatment of CLD was $0.5 \mathrm{mg} / \mathrm{kg} /$ day in two divided doses. This dose was halved after three days and the duration of the tail of the course depended on clinical response.

\section{Serological studies}

Blood samples $(1.5 \mathrm{ml})$ collected immediately before, and eight weeks following the supplementary PRP-T dose were spun and separated. Paired sera were stored at $-70^{\circ} \mathrm{C}$ until batch analysis. Anti-PRP antibody titres were measured at the Centre for Applied Microbiology and Research (Porton Down, UK) by a standardised ELISA protocol. ${ }^{11}$ Antibody avidity was measured by an elution ELISA modified for the Hib assay ${ }^{12}$ and expressed as an avidity index (AI). The lower limit of detection for anti-PRP IgG was $0.07 \mu \mathrm{g} / \mathrm{ml}$ and the minimum measurable AI was 0.06.

\section{Statistical analysis}

In infants with paired samples, antibody levels and avidity indices were log transformed; geometric means for antibody titres (GMTs) and avidity indices (GMAIs) were compared using Student's $t$ test (two sided). Titres of anti-PRP IgG were assessed using the nominal "protective" level of $1.0 \mu \mathrm{g} / \mathrm{ml} .{ }^{13}$ For the purposes of data analysis, sera with unrecordable avidity were assigned a value equal to half the minimum measurable AI.

\section{RESULTS}

Table 1 shows the timing of immunisations and blood samples in the study infants. Table 2 shows gestational age and birth weight, cumulative steroid dose received, anti-PRP antibody concentrations, and avidity indices measured in the serum samples. Infants received up to four courses of dexamethasone (mode number was 1). Median age in days at commencement and cessation of steroid treatment was 12 (range 4-30) and 33 (range 11-227) respectively. Steroid treatment had ceased prior to any immunisations in eight cases, prior to the second Hib dose in a further two cases, and immediately before the fourth Hib dose in cases 2 and 3. Median number of days of steroid treatment was 15 (range 3-153) and cumulative dexamethasone dose administered 3.9 $\mathrm{mg} / \mathrm{kg}$ (range $1.5-25.6 \mathrm{mg} / \mathrm{kg}$ ).

\section{Hib antibodies}

There was no significant increase in antibody titre resulting from the fourth Hib immunisation. Pre- and post-fourth immunisation GMTs were $2.35 \mu \mathrm{g} / \mathrm{ml}$ (95\% CI 0.77 to 7.17 ) and $2.24 \mu \mathrm{g} / \mathrm{ml}$ (95\% CI 0.72 to 7.00$)$ respectively $(\mathrm{p}=0.79)$. Pre-fourth dose serum samples were taken at a median of 43 days (IQR 33-53) after completion of the primary immunisation course, and provided an adequate measure of antibody response to the three dose schedule. Following three doses, six of the 12 infants had already achieved an antibody titre $>1.0$ $\mu \mathrm{g} / \mathrm{ml}$, the level considered to indicate long term protection against $H$ influenzae, ${ }^{13}$ and all five available post-booster titres remained protective following the fourth Hib dose. The six infants with antibody levels less than $1.0 \mu \mathrm{g} / \mathrm{ml}$ following primary immunisation failed to achieve levels $>1.0 \mu \mathrm{g} / \mathrm{ml}$ after a fourth immunisation.

\section{PRP-specific IgG avidity}

There was a significant increase in avidity following the fourth immunisation. GMAI pre-fourth Hib dose was 0.076 (95\% CI 0.045 to 0.128 ) and post-fourth dose 0.138 (95\% CI 0.072 to $0.262)(p=0.043)$. Of 11 infants with paired sera, avidity was detectable in seven following the primary schedule and in nine following the fourth Hib dose. Of the six infants with subprotective antibody titres following primary Hib immunisation, four showed measurable avidity after the three doses; this rose to five following the fourth Hib dose. Only two of six infants with subprotective antibody levels following the fourth Hib dose showed an avidity increase following the fourth dose compared with four of five infants with protective antibody levels.

\section{Adverse incidents}

Enquiry for complications was made during parental interview at the time of the post-booster blood sampling. No untoward reaction to the fourth immunisation was reported in any infant.

Table 2 Clinical characteristics and paired serology measurements of study infants

\begin{tabular}{|c|c|c|c|c|c|c|c|}
\hline $\begin{array}{l}\text { Infant } \\
\text { number }\end{array}$ & $\begin{array}{l}\text { Gestation } \\
\text { (completed } \\
\text { weeks) }\end{array}$ & $\begin{array}{l}\text { Birth weight } \\
\text { (g) }\end{array}$ & $\begin{array}{l}\text { Dexamethasone } \\
\text { cumulative dose } \\
\text { (mg/kg) }\end{array}$ & $\begin{array}{l}\text { Pre-4th Hib Ab } \\
\text { ( } \mu \mathrm{g} / \mathrm{ml})\end{array}$ & $\begin{array}{l}\text { Post-4th Hib Ab } \\
(\mu \mathrm{g} / \mathrm{ml})\end{array}$ & $\begin{array}{l}\text { Pre-4th Hib } \\
\text { avidity index }\end{array}$ & $\begin{array}{l}\text { Post-4th Hib } \\
\text { avidity index }\end{array}$ \\
\hline 1 & 24 & 602 & 7.8 & 0.93 & 0.46 & 0.30 & 0.23 \\
\hline 2 & 24 & 810 & 2.9 & 0.21 & 0.49 & $<0.06$ & $<0.06$ \\
\hline 3 & 25 & 550 & 25.6 & 0.35 & 0.63 & 0.23 & 0.23 \\
\hline 4 & 25 & 620 & 2.0 & 0.86 & 0.75 & 0.09 & 0.09 \\
\hline 5 & 25 & 784 & 4.3 & 0.47 & 0.49 & 0.20 & $>1.0$ \\
\hline 6 & 25 & 860 & 2.8 & 0.90 & 0.45 & $<0.06$ & 0.07 \\
\hline 7 & 26 & 770 & 2.3 & 6.14 & 5.55 & $<0.06$ & $<0.06$ \\
\hline 8 & 26 & 850 & 16.5 & 21.59 & 31.1 & 0.06 & 0.08 \\
\hline 9 & 27 & 1040 & 1.5 & 64.91 & 105.91 & $<0.06$ & 0.19 \\
\hline $10 *$ & 27 & 1130 & 3.6 & 7.05 & NA & 0.07 & NA \\
\hline 11 & 27 & 1235 & 7.8 & 17.06 & 9.44 & 0.13 & 0.15 \\
\hline 12 & 29 & 1210 & 4.2 & 3.25 & 1.74 & 0.06 & 0.47 \\
\hline GMT & & & & 2.35 & 2.24 & 0.076 & 0.138 \\
\hline
\end{tabular}

*Not included in GMT calculation as post-booster serology unavailable. 


\section{DISCUSSION}

Extremely preterm infants have a relative lack of maternally derived IgG and a poorly developed innate immune system, ${ }^{14}$ which may render them at increased risk of invasive Hib disease compared with more mature infants. The available literature also suggests a reduced antibody response in preterm infants following immunisation with certain Hib conjugate vaccines. In two small studies of former preterm infants, $53-55 \%$ achieved protective antibody titres $(>1.0 \mu \mathrm{g} / \mathrm{ml})$ following two PRP-OMP doses. ${ }^{56}$ Eighty two per cent of infants in a small cohort of less than 29 weeks gestation given HbOC vaccine (Hib oligosaccharide conjugated with mutant diphtheria protein) at 2, 4, and 6 months achieved satisfactory titres, a similar percentage to a full term infant comparison group..$^{15}$ Immunogenicity after two PRP-T doses was lower in a small group of infants below 31 weeks gestation than in older preterm infants. $^{7}$

There have been two larger studies in the UK of infants born at less than 32 weeks gestation. Of 59 preterm infants given PRP-T at 2, 3, and 4 months, $44 \%$ of dexamethasone treated infants were adequately protected following the primary schedule compared with $88 \%$ of non-steroid treated infants. ${ }^{8}$ A total of 105 infants given three doses of PRP-T via a combined vaccine achieved an extremely low post-primary Hib GMT, and only $21 \%$ attained protective titres. ${ }^{9}$

Variations in protective antibody rates following immunisation may be caused by many factors relating to both intrinsic and extrinsic characteristics of the population. The limited published data for preterm infants suggest that major adverse influences on antibody response to Hib immunisation are extreme prematurity and steroid treatment. The relative impact of these factors on immunogenicity remains to be evaluated by a larger study. It is noteworthy that the six infants in this study with subprotective antibody levels, both after primary immunisation and following a fourth immunisation, were all born at less than 26 weeks gestation. The most premature infants have the highest rates of corticosteroid treatment for CLD, and the impaired antibody response to primary immunisation in these infants might compound vulnerability to invasive Hib disease.

Present clinical practice regarding immunisation of steroid treated infants lacks consensus. One popular pharmacopoeia advises that it may be preferable to delay immunisation in hospital patients until steroid treatment has stopped, and recommends consideration of a fourth "booster" dose at 12 months of age for those receiving steroids at, or shortly before, immunisation commencement. ${ }^{16}$ A postal questionnaire of leading paediatricians in the North West Region of England in 1999 found that nine of 20 respondents were delaying primary immunisation of steroid treated infants until at least a fortnight following cessation of steroid treatment, and two further respondents were already administering a fourth Hib booster dose (M J Robinson, unpublished data).

We had postulated that an additional Hib vaccine dose given to steroid treated infants six weeks following primary immunisations would boost the antibody response if the immunosuppressive effect of dexamethasone had waned by this time. However, the poor antibody response to a supplementary Hib dose implies persistence of steroid immunosuppression beyond 5 months postnatal age. The duration of steroid suppression of immunogenicity is unknown.

Prematurity is the most important identifiable clinical risk factor associated with Hib vaccine failure in children vaccinated during infancy. ${ }^{17}$ Figures suggest that preterm infants may be overrepresented among Hib vaccine failures in an unboostered cohort, and this adds to concerns about an increased risk of vaccine failure based on reduced antibody responses. The low absolute numbers of preterm infants among vaccine failures could indicate protection by herd immunity ${ }^{18}$ or satisfactory protection of the group notwith- standing attenuated antibody responses. Low antibody levels may still be compatible with clinical protection if immunological memory has been induced by immunisation. Preterm infants with low Hib titres after two PRP-T doses show a booster response comparable to term infants when given a third PRP-T at 12 months. ${ }^{7}$ It remains to be shown whether a similar response occurs in steroid treated infants.

Avidity has been used as a proxy marker for the induction of memory following immunisation with Hib conjugate vaccines. Studies in term infants have shown a fold increase in avidity between post-primary levels and 12 months of age, with little further increase following a booster at 12 months. ${ }^{12} 1920 \mathrm{We}$ observed a virtual doubling of antibody avidity in the eight week period following the booster fourth dose. The significant increase in avidity suggests that despite relatively poor antibody responses, the primary immunisation series has resulted in the generation of immunological memory. The magnitude of the avidity rise over this short period suggests a vaccine booster effect.

An additional Hib dose given to recently steroid treated preterm infants six weeks after the primary course does not appear to improve absolute titre of Hib antibody and cannot be currently recommended. The value of a booster Hib dose given to these infants later in infancy remains to be evaluated. The avidity increase observed following the extra Hib dose suggests a qualitative improvement in antibody associated with boosting, and merits further investigation in a larger study. We do not advocate deferring primary immunisation of steroid treated infants, as postponement delays protection of those at-risk infants capable of responding. Half of those in this study and $44 \%$ (of 43 infants) in a previous study ${ }^{8}$ mounted an adequate antibody response to a routine primary Hib immunisation course despite steroid treatment. For nonresponding infants it is conceivable that routine immunisation has conferred protection via higher avidity antibody albeit present in low titre. Further study is required to assess whether extremely preterm infants develop immune memory in the presence of low antibody levels.

\section{ACKNOWLEDGEMENTS}

We are grateful to Carol Thornton and the Centre for Applied Microbiology and Research for performing the serological testing. We thank Dr Stephen Roberts for allowing the study of a patient in his care and Andrew Vail for statistical advice. The considerable advice and critical appraisal of Dr Scott Morris is sincerely appreciated.

\section{Authors' affiliations}

P Clarke, Neonatal Intensive Care Unit, Hope Hospital, Salford, UK P J Powell, Department of Paediatrics, Royal Bolton Hospital, Bolton, UK D Goldblatt, Immunobiology Unit, Institute of Child Health, Guilford Street, London, UK

M J Robinson, Neonatal Intensive Care Unit, Hope Hospital, Salford, UK

\section{REFERENCES}

1 Vadheim CM, Ward Jl. Epidemiology in developed countries. In: Ellis R, Granoff DM, eds. Development and clinical uses of Haemophilus b conjugate vaccines. New York: Marcel Decker, 1994:231-45.

2 Booy R, Hodgson SA, Slack MPE, et al. Invasive Haemophilus influenzae type $b$ disease in the Oxford region (1985-91). Arch Dis Child 1993;69:225-8

3 Slack MPE, Azzopardi HJ, Hargreaves RM, et al. Enhanced surveillance of invasive Haemophilus influenzae disease in England, 1990 to 1996: impact of conjugate vaccines. Pediatr Infect Dis J 1998;17:S204-7.

4 Hargreaves RM, Slack MPE, Howard AJ, et al. Changing patterns of invasive Haemophilus influenzae disease in England and Wales after introduction of the Hib vaccine programme. BMV 1996;312:160-1.

5 Washburn LK, O'Shea TM, Gillis DC, et al. Response to Haemophilus influenzae type $b$ conjugate vaccine in chronically ill premature infants. J Pediatr 1993;123:791-4.

6 Munoz A, Salvador A, Brodsky NL, et al. Antibody response of low birth weight infants to Haemophilus influenzae type $b$ polyribosylribitol phosphate-outer membrane protein conjugate vaccine. Pediatrics 1995:96:216-19. 
7 Kristensen K, Gyhrs A, Lausen B, et al. Antibody response to Haemophilus influenzae type $b$ capsular polysaccharide conjugated to tetanus toxoid in preterm infants. Pediatr Infect Dis J 1996;15:525-9.

8 Robinson MJ, Campbell F, Powell P, et al. Antibody response to accelerated $\mathrm{Hib}$ immunisation in preterm infants receiving dexamethasone for chronic lung disease. Arch Dis Child Fetal Neonatal Ed 1999;80:F69-F71.

9 Slack MH, Schapira D, Thwaites R, et al. Immune response of premature infants to meningococcal serogroup $C$ and combined diphtheria-tetanus toxoids-acellular pertussis-Haemophilus influenzae type $b$ conjugate vaccines. J Infect Dis 2001;184:1617-20.

10 Salisbury DM, Begg NT, eds. Immunisation against infectious disease. London: HMSO, 1996.

11 Phipps DC, West J, Eby R, et al. An ELISA employing a Haemophilus influenzae type b oligosaccharide-human serum albumin conjugate correlates with the radioantigen binding assay. J Immunol Methods 1990;135:121-8.

12 Goldblatt D, Pinto Vaz ARJPM, Miller E. Antibody avidity as a surrogate marker of successful priming by Haemophilus influenzae type $b$ conjugate vaccines following infant immunization. J Infect Dis 1998; 177:1112-15.
13 Käyhty H, Peltola $\mathrm{H}$, Karanko V, et al. The protective level of serum antibodies to the capsular polysaccharide of Haemophilus influenzae type b. J Infect Dis 1983;147:1100.

14 Goldblatt D. Immunisation and the maturation of infant immune responses. Dev Biol Stand 1998:95:125-32.

15 D'Angio CT, Maniscalco WM, Pichichero ME. Immunologic response of extremely premature infants to tetanus, Haemophilus influenzae type $b$, and polio immunizations. Pediatrics 1995;96:18-22.

16 Northern Neonatal Network. Neonatal formulary, 3rd edn. London: BM Books, 2000: 130,243.

17 Heath PT, Booy R, Griffiths $\mathrm{H}$, et al. Clinical and immunological risk factors associated with Haemophilus influenzae type $b$ conjugate vaccine failure in childhood. Clin Infect Dis 2000;31:973-80.

18 Heath PT, Booy R, Azzopardi HJ, et al. Antibody concentration and clinical protection after Hib conjugate vaccination in the United Kingdom. JAMA 2000;284:2334-40.

19 Goldblatt D, Richmond P, Millard E, et al. The induction of immunologic memory after vaccination with Haemophilus influenzae type $b$ conjugate and acellular pertussis-containing diphtheria, tetanus, and pertussis vaccine combination. J Infect Dis 1999;180:538-41.

20 Poolman J, Kaufhold A, De Grave D, et al. Clinical relevance of lower Hib response in DTPa-based combination vaccines. Vaccine $2001 ; 19: 2280-5$.

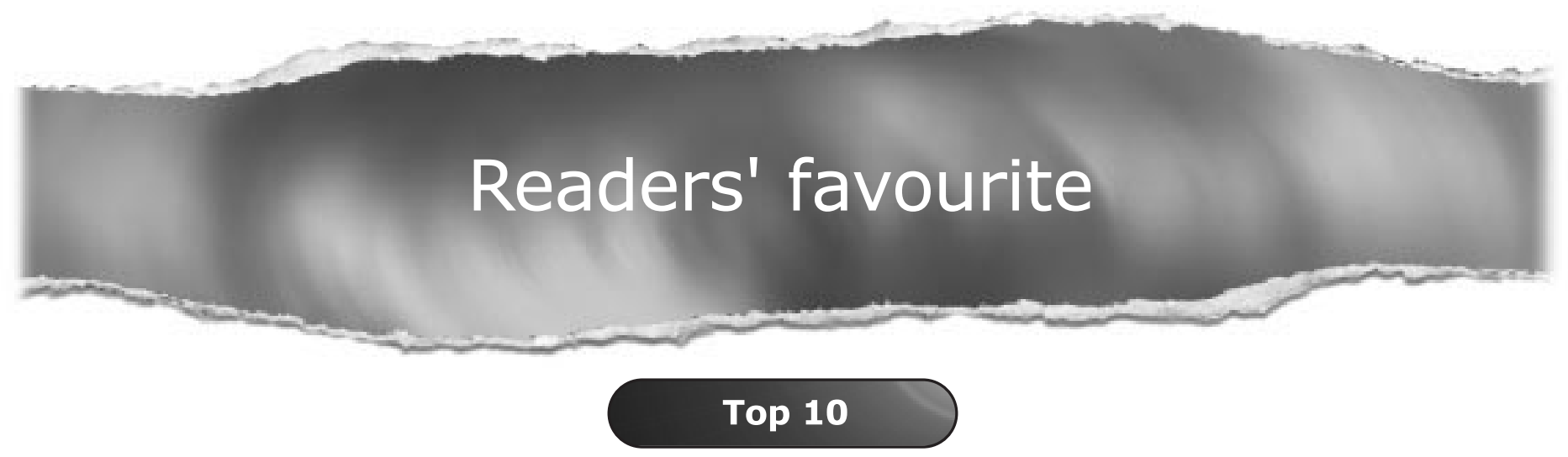

Click on the "Top 10" button on the homepage to see which are the best read articles each month

www.archdischild.com 